\title{
SWATCH: A StepWise AdapTive Clustering Hierarchy in Wireless Sensor Networks
}

\author{
Quanhong Wang ${ }^{1}$, Kenan $\mathrm{Xu}^{1}$, Hossam Hassanein ${ }^{2}$, and Glen Takahara ${ }^{3}$ \\ ${ }^{1}$ Department of Electrical and Computer Engineering, Queen's University, Canada \\ $\{1 \mathrm{qw}, 1 \mathrm{kx}\} @ \mathrm{qlink} . q u e e n s u . c a$ \\ 2 School of Computing, Queen's University, Canada \\ hossam acs.queensu.ca \\ ${ }^{3}$ Department of Mathematics and Statistics, Queen's University, Canada \\ takahara@mast.queensu.ca
}

\begin{abstract}
Distributed clustering techniques are considered effective and practical for power-saving in Wireless Sensor Networks (WSNs). In this paper, we propose a novel cluster formation scheme in WSNs, called StepWise AdapTive Clustering Hierarchy (SWATCH). SWATCH aims to solve the ClusterHead $(\mathrm{CH})$ number uncertainty problem common to existing distributed clustering schemes, while keeping the desirable properties of self-organization, simplicity and dynamic adaptation. We develop a two-tier hierarchical Markov chain model to track the operation of SWATCH. Based on this model, we derive formulations of the statistical properties of our proposed scheme. Numerical results verify the design objectives in that the number of selected clusterheads highly conforms to the optimal value.
\end{abstract}

\section{Introduction}

Communication cost has been shown to be the major source of energy dissipation in Wireless Sensor Networks (WSNs) [1]. Clustering is an effective framework under which different energy saving techniques, such as energy-saving MAC and local data processing, can be easily adopted. With a clustered structure, the entire network is partitioned into disjoint clusters. Each cluster consists of one ClusterHead $(\mathrm{CH})$ and multiple Member Nodes (MNs). Forming the virtual backbone of the network, the $\mathrm{CHs}$ collect data from MNs and relay the processed data, e.g., aggregated data [2] to the base station.

Although the optimal number of $\mathrm{CHs}$ has been proven to exist for WSNs using certain clustering scheme [2,3], clustering schemes fail to guarantee the optimal number of $\mathrm{CHs}$ that will be elected in a dynamical basis. For example, LEACH [2] achieves energy saving by having the network dynamically partitioned into multiple clusters and local data processing in each of them. However, due to the randomness in the $\mathrm{CH}$ selection algorithm, the number of $\mathrm{CHs}$ is not guaranteed to be equal to the expected optimal value (according to our investigation, the percentage of time that the number of $\mathrm{CHs}$ is equal to the optimal value is observed to be less than $20 \%$, see Fig. 1.) In the worse case, when one $\mathrm{CH}$ serves the whole network, its energy would drain rapidly, and fairness is severely affected. 


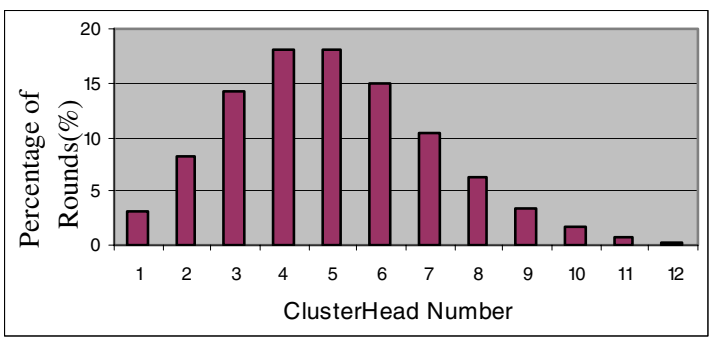

Fig. 1. $\mathrm{CH}$ Number Distribution of LEACH (100 nodes, Target $\mathrm{CH}$ Number $=5$. The percentage of $\mathrm{CH}$ number greater than 12 is negligible)

In this paper, we propose a novel single hop clustering scheme called StepWise AdapTive Clustering Hierarchy (SWATCH). SWATCH relieves the $\mathrm{CH}$ number variability problem by employing stepwise $\mathrm{CH}$ selection in two stages. It is a dynamic and straightforward scheme as LEACH. However, instead of selecting all CHs in one step, SWATCH splits the selection phase into an initial selection stage and an add-on selection stage. The initial selection is similar to $\mathrm{LEACH}$. However, if the number of $\mathrm{CHs}$ in the initial selection is below a pre-determined target, the add-on selection will be invoked and will continue until an acceptable number of $\mathrm{CHs}$ have been selected. As a result, the number of $\mathrm{CHs}$ selected in each round tends to congregate in a narrow range around the optimal value. In other words, the variance of the number of $\mathrm{CHs}$ in each round is decreased. In order to evaluate the performance of SWATCH, we develop a hierarchical Markov chain model to track the behavior of the system. Numerical results verify our design objective in that the number of selected $\mathrm{CHs}$ highly conforms to the optimal value. Based on the results in $[2,3]$, which show that an optimal number of $\mathrm{CHs}$ can greatly reduce the communication energy, we argue that SWATCH is energy efficient and fair.

The remainder of this paper is organized as follows. In section 2, the SWATCH scheme is presented. In section 3, the performance of SWATCH is evaluated by comparing to LEACH scheme.

\section{SWATCH Description}

\subsection{Preliminaries}

We make the following assumptions about the network and communication models. We assume a static network consisting of $\mathrm{N}$ identical stationary nodes, which are distributed randomly in a 2-dimensional sensing field (to fulfill a constant sensing task). The data is pumped out proactively at each node. A fixed base station is located far from the network to gather the collective information.

We assume that all nodes are capable of adjusting their transmission power according to transmission distance, and they can transmit with enough power to reach the BS and other nodes in the network if necessary. In order to conserve energy, each member node sends its data to its associated $\mathrm{CH}$, and the $\mathrm{CH}$ processes the data and sends the aggregated information to the base station. The $\mathrm{CH}$ also performs local coordination in the cluster as in [2,3]. Moreover we assume error-free communication as in [3]. 


\subsection{SWATCH Scheme}

In SWATCH, $\mathrm{CH}$ selection and cluster formation is on round basis. The $\mathrm{CH}$ Selection Period (CHSP) is split into two stages, namely, the $\mathrm{CH}$ Initial Selection Stage (CHISS) and the $\mathrm{CH}$ Add-on Selection Stage (CHASS). In the CHISS, each $\mathrm{CH}$ candidate independently makes a decision to be a $\mathrm{CH}$ using the probability $p_{\text {Init }}$ $\left(p_{\text {Init }}=\left(P_{\text {Init }} / i\right)^{*} N\right)$, where $N$ is the total number of nodes in the system, $P_{\text {Init }}$ is the initial selection probability in a round, and $i$ is the number of $\mathrm{CH}$ candidates at the beginning of the round). If the number of $\mathrm{CHs}$ in the CHISS reaches or exceeds a predetermined target value $T_{A}$, the CHSP terminates. Otherwise, the CHASS will be invoked. In the CHASS, all remaining $\mathrm{CH}$ candidates take part in the $\mathrm{CH}$ add-on procedure and make a decision to be $\mathrm{CH}$ based on the probability $p_{\text {Add }_{k}}$ ( $\left.p_{\text {Add }}=\left(P_{\text {Add }} / k\right)^{*} N\right)$, where $P_{\text {Add }}$ is the add-on selection probability and $k$ is the number of $\mathrm{CH}$ candidates at beginning of the CHASS). Add-on selection is invoked repeatedly until the total number of $\mathrm{CHs}$ in the current round is equal to or greater than $T_{A}$. A timeline showing the rounds and stages is depicted in Fig. 2.

In order to speed up the $\mathrm{CH}$ selection procedure and avoid unnecessary $\mathrm{CH}$ selection due to a small number of $\mathrm{CH}$ candidates, we introduce the CHSP-End threshold $T_{E}\left(T_{E}>T_{A}\right)$. When the number of $\mathrm{CH}$ candidates is less than or equal to $T_{E}$, all $\mathrm{CH}$ candidates will become CHs automatically; otherwise, the normal CHISS is invoked.

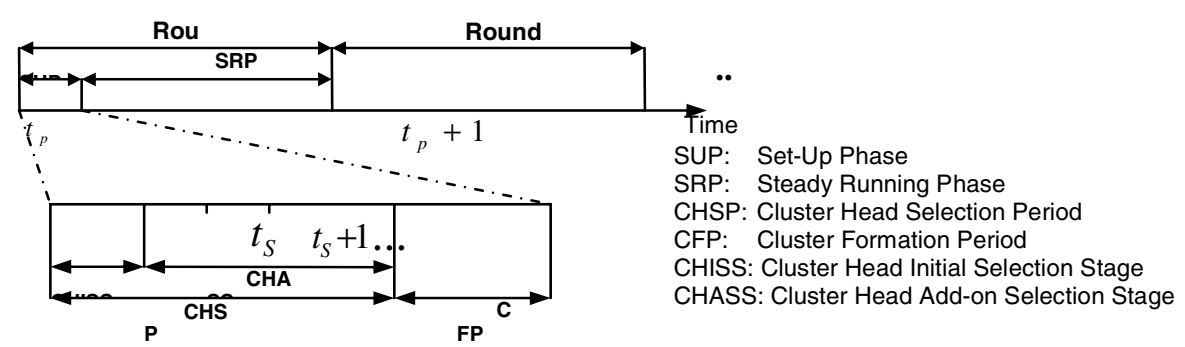

Fig. 2. Illustration of SWATCH Timeline

\section{Performance Evaluation}

To evaluate the effectiveness of SWATCH, we develop a hierarchical Markov chain model, consisting of a Primary Markov Chain (PMC) and a Secondary Markov Chain (SMC). The PMC is used to describe the $\mathrm{CH}$ selection procedure from round to round, while the SMC is used to describe the $\mathrm{CH}$ selection behavior during the CHASS. The model is omitted due to the space limitation. Based on it, we calculate the expectation and Coefficient of Variance (C.O.V) of the number of CHs, as well as the average extra number of $\mathrm{CH}$ selection steps. 
Fig. 3 illustrates the results for the C.O.V of the number of $\mathrm{CHs}$ using both LEACH and SWATCH for the same WSN with different target $\mathrm{CH}$ numbers. In all the cases, SWATCH produces much smaller C.O.V. than LEACH. It implies that SWATCH can ensure a more stable number of CHs.

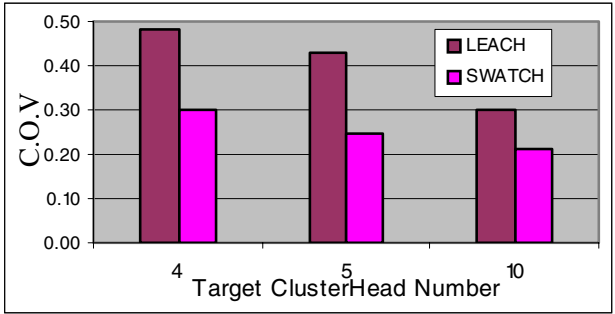

Fig. 3. C.O.V of ClusterHead Number (100 nodes, target values $=4,5,10)$

SWATCH addresses the $\mathrm{CH}$ number uncertainty problem explicitly by exploiting a two-stage $\mathrm{CH}$ selection algorithm. Since it has been earlier shown that there exists an optimal value for individual WSNs, which leads to the minimum energy dissipation, we argue that SWATCH will provide higher energy efficiency than the existing distributed schemes.

\section{References}

1. V. Raghunathan, C. Schurgers, S. Park and Mani B. Srivastava, "Energy-aware Wireless Microsensor Networks", IEEE Signal Processing Magazine, Vol. 19, Issue 2, pp. 40-50, March 2002.

2. W. B. Heinzelman, A. P. Chandrakasan, H. Balakrishnan, "An application-specific protocol architecture for Wireless Microsensor Networks", IEEE Tran. On Wireless Communications, Vol. 1, No. 4, pp.660-670, Oct. 2002.

3. S. Bandyopadhyay and E. J. Coyle, "An energy efficient hierarchical clustering algorithm for Wireless Sensor Networks”, IEEE INFOCOM 2003, USA, Mar. - Apr. 2003. 